\title{
Interindividual variation in gene expression responses and metabolite formation in acetaminophen-exposed primary human hepatocytes
}

\author{
Marlon J. A. Jetten ${ }^{1} \cdot$ Ainhoa Ruiz-Aracama ${ }^{2} \cdot$ Maarten L. J. Coonen $^{1} \cdot$ \\ Sandra M. Claessen ${ }^{1}$ Marcel H. M. van Herwijnen ${ }^{1}$ - Arjen Lommen ${ }^{2}$. \\ Joost H. M. van Delft ${ }^{1} \cdot$ Ad A. C. M. Peijnenburg $^{2} \cdot$ Jos C. S. Kleinjans $^{1}$
}

Received: 6 January 2015 / Accepted: 3 June 2015 / Published online: 24 June 2015

(C) The Author(s) 2015. This article is published with open access at Springerlink.com

\begin{abstract}
Acetaminophen (APAP) is a readily available over-the-counter drug and is one of the most commonly used analgesics/antipyretics worldwide. Large interindividual variation in susceptibility toward APAP-induced liver failure has been reported. However, the exact underlying factors causing this variability in susceptibility are still largely unknown. The aim of this study was to better understand this variability in response to APAP by evaluating interindividual differences in gene expression changes and APAP metabolite formation in primary human hepatocytes $(\mathrm{PHH})$ from several donors $(n=5)$ exposed in vitro to a non-toxic to toxic APAP dose range. To evaluate interindividual variation, gene expression data/levels of metabolites were plotted against APAP dose/donor. The correlation in APAP dose response between donors was calculated by comparing data points from one donor to the data points of all other donors using a Pearson-based correlation analysis. From that, a correlation score/donor for each gene/metabolite was defined, representing the similarity of the omics response to APAP in PHH of a particular donor to all other donors. The top $1 \%$ highest variable genes were selected for further evaluation using gene set overrepresentation analysis. The biological processes in which the genes with
\end{abstract}

Electronic supplementary material The online version of this article (doi:10.1007/s00204-015-1545-2) contains supplementary material, which is available to authorized users.

Marlon J. A. Jetten

marlon.jetten@maastrichtuniversity.nl

1 Department of Toxicogenomics, Maastricht University, Universiteitssingel 50, Room 4.112 UNS 50, 6229 ER Maastricht, The Netherlands

2 RIKILT, Institute of Food Safety, Wageningen UR, PO Box 230, 6700 AE Wageningen, The Netherlands high interindividual variation in expression were involved include liver regeneration, inflammatory responses, mitochondrial stress responses, hepatocarcinogenesis, cell cycle, and drug efficacy. Additionally, the interindividual variation in the expression of these genes could be associated with the variability in expression levels of hydroxyl/methoxy-APAP and $\mathrm{C} 8 \mathrm{H} 13 \mathrm{O} 5 \mathrm{~N}-\mathrm{APAP}$-glucuronide. The before-mentioned metabolites or their derivatives have also been reported in blood of humans exposed to therapeutic APAP doses. Possibly these findings can contribute to elucidating the causative factors of interindividual susceptibility toward APAP.

Keywords Interindividual variation - Primary human hepatocytes · DNA methylation - Gene expression · Aflatoxin b1 $\cdot$ Benzo(a)pyrene

\section{Introduction}

Acetaminophen (APAP) is one of the most commonly used analgesics/antipyretics worldwide. Thereby, it is a readily available over-the-counter drug. Apart from its beneficial effects as a pharmaceutical, APAP is also the most common cause of acute liver toxicity in Europe and the USA (Lee 2007). Cytochrome P450 enzymes in the liver metabolize APAP to the oxidative metabolite $N$-acetyl- $p$-benzoquinone imine (NAPQI), which is thought to cause the toxic effects of APAP through protein adduct formation, leading to oxidative stress and finally liver damage (Dahlin et al. 1984a). The molecular mechanism behind further progression of APAP toxicity still is not fully elucidated; the involvement of multiple mechanisms of toxicity, like inflammatory responses and oxidative stress, has been suggested (Jaeschke et al. 2012).

The main body of our knowledge on the toxicological, or more specific hepatotoxic, mechanisms of compounds, 
including APAP, is based on data collected from studies using animal models (Hinson et al. 2010). However, increasing criticism on the usability and applicability of animal data to the human situation has developed over the last years (Greek and Menache 2013). Therefore, there is a growing need for human in vitro models such as hepatic cell lines, liver slices, or primary hepatocytes cultures, to facilitate human-based research.

Large interindividual differences in response to xenobiotic exposure between humans have been documented (Court et al. 2001). In the domains of toxicology/pharmacology, many attempts are being undertaken to gain a better understanding of the factors that are causative of this interindividual variation. Environmental factors as well as genetic factors have been proposed to contribute to the variation in drug responses between human individuals. Over the last decade, with the rise of the whole genome omics techniques, it has become feasible to perform more complete/in-depth analysis of the genomic components contributing to interindividual variation in the human population (Berg 2014).

Also, the metabolism of APAP is known to show large interindividual variability (Court et al. 2001). Genetic factors including many biotransformation-related genes, such as UDP-glucuronosyltransferases and cytochrome P450 enzymes, have been suggested to be causative of the variation in APAP-induced adverse effects observed between individuals (Court et al. 2001; Fisher et al. 2000; Polasek et al. 2006; Yasar et al. 2013). While some individuals seem to be able to endure APAP doses considerably exceeding the recommended maximal daily dose, others are at risk of liver toxicity due to APAP much closer to the recommended dose window (Sabate et al. 2011). The relatively high frequency of unintentional overdosing has recently led the FDA to adjusting recommendations on safe APAP dosage use by lowering the maximal therapeutic daily dose and decreasing the single dose units of APAP (Turkoski 2010).

However, not only interindividual differences do occur at supra-therapeutic doses, but also (sub-) therapeutic doses of APAP have been shown to cause interindividual variation in APAP metabolite levels, as well as in mRNA and miRNA expression levels (Jetten et al. 2012). In the same study, regulation of biological processes known to be related to the liver toxicity response after APAP overdosing could be detected at lower and supposedly non-toxic doses.

In this study, we aim at investigating the interindividual differences in response to a non-toxic to toxic APAP dose range, using an in vitro cell model consisting of primary human hepatocytes of several donors. The interindividual differences in APAP metabolite formation and gene expression responses were considered and compared in an attempt to pinpoint the factors that could be causative of interindividual variation in APAP metabolism.

\section{Materials and methods}

\section{Cell culture and treatment}

\section{Primary human hepatocytes}

Cryopreserved PHH of five individuals (see Supplementary Table 1 for donor demographics) were purchased from Life Technologies (Gibco). Cells were cultured in 12 -well plates in a collagen sandwich, according to the supplier's protocol (Invitrogen 2009). The following culture media were used: cryopreserved hepatocyte recovery medium (CHRM, Gibco) for thawing, William's medium $\mathrm{E}$ (WME) + Glutamax (Gibco) substituted with $10 \%$ FCS (Gibco), $0.02 \%$ penicillin/streptomycin (Gibco), and $0.1 \mathrm{U} / \mathrm{ml}$ insulin (Invitrogen) for seeding/attaching and WME + Glutamax substituted with $0.02 \%$ Pen/Strep, $0.1 \mathrm{U} / \mathrm{ml}$ insulin, and $0.02 \mathrm{mg} / \mathrm{ml}$ hydrocortisone (SigmaAldrich) for culturing/exposure. After thawing, viability of the cells was checked by a trypan blue (CAS no. 72-57-1, Sigma-Aldrich) exclusion test as instructed in the supplier's protocol (Invitrogen 2009). All viability scores were in accordance with those listed by the supplier. Hepatocytes were exposed for $24 \mathrm{~h}$ to $0,0.2,2$, or $10 \mathrm{mM}$ APAP (CAS no. 103-90-2, Sigma-Aldrich) dissolved in culture medium. The doses, representing no observed effect level (NOEL), lowest observed effect level (LOEL $=$ nontoxic), and toxic dose, respectively, were chosen based on the available literature (Bannwarth et al. 2001; Borin and Ayres 1989; Critchley et al. 2005; Douglas et al. 1996; Kamali 1993; Kienhuis et al. 2009; Portolés et al. 2003; Rygnestad et al. 2000; Tan and Graudins 2006; Yin et al. 2001).

\section{Transcriptomic sample preparation}

\section{Total RNA isolation}

QIAzol (0.5 ml, QIAGEN) was used to isolate total RNA from all samples according to the manufacturer's protocol. RNA purification was performed with the miRNeasy Mini Kit (Qiagen) as instructed by the manufacturer. Next, the integrity of the RNA was checked with the Bioanalyzer 2100 (Agilent).

\section{cDNA preparation/hybridization}

From an input of 250 ng RNA, cDNA targets were prepared using the Affymetrix protocol. The procedures as recommended by the manufacturer were applied to hybridize the samples to Affymetrix GeneChip Human Genome U133A plus 2 GeneChip arrays. GeneChips were washed and stained after hybridization with a fluidics station 
(Affymetrix) and scanned with a GeneArray scanner (Affymetrix). The samples from donor 1 exposed to $10 \mathrm{mM}$ APAP did not pass quality control and were therefore excluded from further analyses.

\section{Transcriptomic data analysis}

The CEL files retrieved in the previous step were subjected to an overall quality control, using arrayanalysis.org, and all arrays were of high quality (Eijssen et al. 2013). Subsequently, data were RMA-normalized and re-annotated using BrainArray's EntrezGene customCDF_V15.1 (Dai et al. 2005; Lim et al. 2012). Probes with low signal-tonoise ratio (average expression $<6$ ) were excluded from further analyses as a data-cleanup step.

\section{Metabolomic sample preparation}

Culture medium from the cells was collected after $24 \mathrm{~h}$ and stored at $-80{ }^{\circ} \mathrm{C}$ until extraction. To extract the metabolites, $6 \mathrm{ml}$ of ice-cold acetone was added to $1.5 \mathrm{ml}$ of medium in a 10-ml glass tube. The solution was vortexed for $30 \mathrm{~s}$, kept on ice for $12 \mathrm{~min}$, and then centrifuged for $15 \mathrm{~min}$ at $2800 \mathrm{rpm}$ at $4{ }^{\circ} \mathrm{C}$. The supernatant was transferred into a 10-ml glass tube and dried under nitrogen. To concentrate the semi-polar metabolites contained in the medium, SPE C18 columns (C18, $500 \mathrm{mg}, 3 \mathrm{ml}$, BondElut, VARIAN) were used. The SPE C18 column was conditioned by running it with methanol including $0.5 \%$ of formic acid ( $1 \mathrm{ml}$ twice) and MilliQ including $0.5 \%$ of formic acid ( $1 \mathrm{ml}$ twice). Once the dried pellet was resuspended in $1 \mathrm{ml}$ of MilliQ (with $0.5 \%$ formic acid), it was applied to the column. The column was then washed twice with $1 \mathrm{ml}$ of MilliQ, after which the components of interest were eluted with $1 \mathrm{ml}$ of methanol and dried under nitrogen. For U-HPLC-Orbitrap analysis, the dried polar fraction was re-suspended in $400 \mu \mathrm{l}$ of MilliQ with $0.1 \%$ formic acid.

\section{U-HPLC-Orbitrap MS analysis}

Experimental setups and procedures as described before have been used with some slight modifications as defined below (Lommen 2009; Lommen et al. 2011; Ruiz-Aracama et al. 2011). The gradient was similar to the one used in Jetten et al. (2012) with small modifications. The initial eluent composition, $100 \% \mathrm{~A}$, was changed to $85 \% \mathrm{~A}$ and $15 \% \mathrm{~B}$ in 15 min. Afterward, the composition of B was increased to $30 \%$ in $10 \mathrm{~min}$ and subsequently increased in $3 \mathrm{~min}$ to $90 \%$, remaining at this composition for $5 \mathrm{~min}$ prior to the next injection. A capillary temperature of $250{ }^{\circ} \mathrm{C}$ with a sheath and auxiliary gas flow of 19 and 7 arbitrary units were used, respectively.

\section{Metabolomic data analysis}

Visual inspection of the three technical replicates of each sample showed a high degree of reproducibility. All MS data were preprocessed and aligned using the in-house developed program, metAlign (Lommen 2009). A targeted search for the metabolites of APAP previously described in Jetten et al. (2012) was executed. For targeted analyses, Search LCMS, an add-on tool for metAlign, was used (Lommen 2009). Briefly, a list of masses of interest was composed based on our previous in vivo study with human volunteers (Jetten et al. 2012) and some further data available from mice (Chen et al. 2008a). This list was loaded into Search LCMS, which returned the amplitudes of the masses of interest.

U-HPLC-Orbitrap MS data were preprocessed as described in previous papers (Lommen 2009; Lommen et al. 2011) to obtain ultra-precise (sub-ppm) mass data (calibration using internal masses and PEG200, PEG300, PEG600 as external masses). Metabolites were considered to be present when retention times were analogous to earlier experiments and average accurate masses were below $\pm 3 \mathrm{ppm}$; nearly all average masses of metabolites were within $1 \mathrm{ppm}$. For some metabolites previously not found, including hydroxy-APAP, methoxy-APAP, and 3,3'-biacetaminophen, retention times were related to those derived from the literature if possible (Chen et al. 2008a; Jetten et al. 2012). Metabolite expression levels for methoxy-APAP-glucuronide-1/2 and hydroxy-APAP-glutathione in samples of donor 1 exposed to $10 \mathrm{mM}$ APAP could unfortunately not be determined due to technical issues.

\section{Metabolite visualization}

To create a metabolic map based on available literature, a pathway visualization tool, PathVisio, was used (Chen et al. 2008b; Daykin et al. 2002; van Iersel et al. 2008). LC-MS data were visualized for each donor and per dose (corrected for control levels, $0 \mathrm{mM}$ APAP). Log-transformation of the data resulted in range with a minimum of 0 and a maximum of 5 .

\section{Data integration and visualization; interindividual variation}

Expression data ( $\log 2$-scaled intensities) of all genes passing the selection as described under 'transcriptomic data analysis' and levels of all of the identified metabolites were plotted against APAP dose per donor $(X$-axis: dose $0,0.2,2$, and $10 \mathrm{mM}$ APAP; $Y$-axis: $\log$-scaled gene expression/metabolite levels; line: donor, $n=5$ ) using $\mathrm{R}$ 2.15.3 (R Core Team 2013). For clarification purposes, a 
representative plot is provided in Supplementary Figure 1. To estimate the correlation in APAP dose response between donors, data points from one donor were compared to the data points of all other donors using a Pearson-based correlation analysis, which resulted in the following comparisons: D1-D2, D1-D3, D1-D4, D1-D5, D2-D3, D2-D4, D2-D5, D3-D4, D3-D5, and D4-D5. Then, the absolute correlation coefficients of each donor were summed to generate an arbitrary correlation score per donor for each gene/ metabolite (Score D1, Score D2, Score D3, Score D4, and Score D5; see supplementary Figure 1). This 'score' now represents the similarity of a particular donor to all other donors in expression response for a particular gene/metabolite following APAP exposure; the donor with the lowest score is most aberrant from all other donors (showed the least correlation with the other donors). In order to select the most variable genes between donors, standard deviations (SD) of the donor scores per gene/metabolite were calculated and ranked. The top $1 \%$ ranked genes (score $S D>2.52$ ) were selected for further analysis, since these exhibit the most interdonor variability (Table 1 ).

To enable biological interpretation, an overrepresentation analysis was performed on this set of variable genes, using the overrepresentation module of ConsensusPathDB (Kamburov et al. 2013). A background list consisting of all genes passing the selection as described under 'transcriptomic data analysis' was used in this analysis.

Subsequently, the correlation score matrices (see top left corner supplementary Figure 1) created for each of the top $1 \%$ highly variable genes and all metabolites were used to find gene expression profiles that match metabolite profiles on an individual level. To do so, the interdonor correlations for all highly variable genes were correlated with the interdonor correlations from all metabolites (Pearson). A cutoff of $>0.7$ was used to define genes of which the difference in expression levels mimicked the difference in the metabolite levels between donors. The results of this analysis are summarized in supplementary Table 3 .

To define genes related to mitochondrial processes, the top $1 \%$ variable genes were compared to the mitochondrial reference gene set from MITOP2. This is a database which provides a list of human mitochondrial proteins linked to their gene names found through computational prediction of signaling sequences, but also includes results from proteome mapping, mutant screening, expression profiling, protein-protein interaction, and cellular sub-localization studies (Elstner et al. 2009). The MiMI plugin for Cytoscape was used to generate a network for all mitochondrial-related genes based on the nearest neighbor analysis (Fig. 3; Gao et al. 2009). Only the nearest neighbors shared by more than one of the mitochondrial-related genes were taken into consideration.

\section{Results}

\section{Transcriptomics}

Just over 10,000 genes were screened for interindividual variation in their responses toward APAP exposure by correlating their expression over dose. Standard deviations of these correlation scores showed a normal distribution. To assure that only the most variable genes were used for further analyses, and a short list was created of the top $1 \%$ most variable genes (see Table $1, n=99, \mathrm{SD}>2.52$ ).

To define the functionality of the variable genes, an overrepresentation analysis was performed, i.e., a network consisting of the biological pathways containing the genes with the highest variability between donors in response to APAP exposure was defined (see Fig. 1). This overrepresentation network could be broken down into several parts; one large component appeared constructed of:

- A large cluster with toll-like receptors (TLR), c-Jun $\mathrm{N}$-terminal kinases (JNK), nuclear factor (NF)- $\mathrm{KB}$, interleukin (IL)-1, p38, and cyclin D1-related pathways (encircled with striped line).

- A smaller cluster around p75 neurotrophin receptor (p75 NTR) (encircled with dotted line).

- Two more components, involved in 'Wnt-signaling pathway and pluripotency' and 'BTG (B cell translocation gene) family proteins and cell cycle regulation.'

Additional parts consisted of components separated from the large cluster on 'leukotriene metabolism,' 'biosynthesis of unsaturated fatty acids,' 'amino sugar and nucleotide sugar metabolism,' 'retinoic acid-inducible gene 1 (RIG-I), and melanoma differentiation-associated protein 5 (MDA5)-mediated induction of interferon (IFN)-alpha-beta.'

A further network based on mitochondrial-related genes form the top $1 \%$ highly variable genes was created using next neighbor analysis in Cytoscape Fig. 2. This figure shows that transcription factors are the main binding element in the response of mitochondrial-related genes, showing high interindividual variation in gene expression response after APAP exposure.

\section{Metabolomics}

A broad spectrum of metabolites was measured in the medium, as shown in Supplementary Table 3 and Figure 3. In general, the variation between individuals was lower with respect to metabolite levels when compared to the variation in gene expression levels. To define how the variability between donors in gene expression is related to 
Table 1 List of the top $1 \%$ most variable genes based on Pearson correlation analysis

\begin{tabular}{|c|c|c|}
\hline EntrezGeneID & Gene name & Functional description according to GeneCards \\
\hline 92 & ACVR2A & Kinase receptor \\
\hline 513 & $A T P 5 D$ & Subunit of mitochondrial ATP synthase \\
\hline 595 & CCND1 & Cyclin family \\
\hline 617 & $B C S 1 L$ & Complex III of the mitochondrial respiratory chain \\
\hline 988 & CDC5L & Cell cycle regulator important for G2/M transition \\
\hline 1545 & CYP1B1 & Cytochrome P450 superfamily \\
\hline 1611 & DAP & Mediator of programmed cell death \\
\hline 2669 & GEM & GTP-binding proteins, receptor-mediated signal transduction \\
\hline 2766 & GMPR & NADPH-dependent reductive deamination of GMP to IMP \\
\hline 3276 & PRMT1 & Methyltransferase \\
\hline 4302 & MLLT6 & Myeloid/lymphoid or mixed-lineage leukemia \\
\hline 4615 & MYD88 & Myeloid differentiation primary response \\
\hline 5201 & PFDN1 & Member of the prefolding beta subunit family \\
\hline 5287 & PIK3C2B & $\begin{array}{l}\text { PI3-kinases play roles in signaling pathways involved in cell proliferation, oncogenic transformation, cell } \\
\text { survival, cell migration, and intracellular protein trafficking }\end{array}$ \\
\hline 5300 & PIN1 & $\begin{array}{l}\text { Regulation of cell growth, genotoxic, and other stress responses, the immune response, induction and mainte- } \\
\text { nance of pluripotency, germ cell development, neuronal differentiation, and survival }\end{array}$ \\
\hline 5523 & PPP2R3A & Negative control of cell growth and division \\
\hline 5550 & PREP & Maturation and degradation of peptide hormones and neuropeptides \\
\hline 5584 & PRKCI & $\begin{array}{l}\text { Protective role against apoptotic stimuli, is involved in NF-kappa-B activation, cell survival, differentiation, } \\
\text { and polarity and contributes to the regulation of microtubule dynamics in the early secretory pathway }\end{array}$ \\
\hline 5696 & PSMB8 & Apoptosis, may be involved in the inflammatory response pathway \\
\hline 5699 & PSMB10 & Involved in antigen processing to generate class I-binding peptides \\
\hline 5796 & PTPRK & Cell growth, differentiation, mitotic cycle, and oncogenic transformation \\
\hline 6612 & SUMO3 & $\begin{array}{l}\text { Posttranslationally modify numerous cellular proteins and affect their metabolism and function, such as } \\
\text { nuclear transport, transcriptional regulation, apoptosis, and protein stability }\end{array}$ \\
\hline 6942 & TCF20 & $\begin{array}{l}\text { Stimulates the activity of various transcriptional activators such as JUN, SP1, PAX6, and ETS1, suggesting a } \\
\text { function as a co-activator }\end{array}$ \\
\hline 7264 & TSTA3 & Cell-cell interactions, including cell-cell recognition; in cell-matrix interactions; in detoxification processes \\
\hline 7572 & ZNF24 & Transcription repressor activity \\
\hline 7965 & AIMP2 & Functions as a pro-apoptotic factor \\
\hline 8270 & LAGE3 & ??? \\
\hline 8310 & $A C O X 3$ & Desaturation of 2-methyl branched fatty acids in peroxisomes \\
\hline 8985 & PLOD3 & Hydroxylation of lysyl residues in collagen-like peptides \\
\hline 9343 & EFTUD2 & A component of the spliceosome complex which processes precursor mRNAs to produce mature mRNAs \\
\hline 9361 & LONP1 & $\begin{array}{l}\text { Mediates the selective degradation of misfolded, unassembled, or oxidatively damaged polypeptides in the } \\
\text { mitochondrial matrix assembly of inner membrane protein complexes and participates in the regulation of } \\
\text { mitochondrial gene expression and maintenance of the integrity of the mitochondrial genome }\end{array}$ \\
\hline 9470 & EIF4E2 & $\begin{array}{l}\text { EIF4E2 gene promoter protein synthesis and facilitates ribosome binding by inducing the unwinding of the } \\
\text { mRNA secondary structures }\end{array}$ \\
\hline 10093 & ARPC4 & $\begin{array}{l}\text { Regulation of actin polymerization and together with an activating nucleation-promoting factor (NPF) medi- } \\
\text { ates the formation of branched actin networks }\end{array}$ \\
\hline 10189 & THOC4 & $\begin{array}{l}\text { Molecular chaperone. It is thought to regulate dimerization, DNA binding, and transcriptional activity of basic } \\
\text { region-leucine zipper (bZIP) proteins }\end{array}$ \\
\hline 10313 & RTN3 & Involved in membrane trafficking in the early secretory pathway \\
\hline 10422 & UBAC1 & $\begin{array}{l}\text { Required for poly-ubiquitination and proteasome-mediated degradation of CDKN1B during G1 phase of the } \\
\text { cell cycle }\end{array}$ \\
\hline 10598 & AHSA1 & May affect a step in the endoplasmic reticulum to Golgi trafficking \\
\hline 10807 & SDCCAG3 & May be involved in modulation of TNF response \\
\hline 10899 & JTB & Required for normal cytokinesis during mitosis. Plays a role in the regulation of cell proliferation \\
\hline
\end{tabular}


Table 1 continued

\begin{tabular}{|c|c|c|}
\hline EntrezGeneID & Gene name & Functional description according to GeneCards \\
\hline 11068 & CYB561D2 & $\begin{array}{l}\text { Acting as an ubiquitin-conjugating enzyme, involved in the regulation of exit from mitosis, cell cycle, protein, } \\
\text { ubiquitin-dependent proteolysis, electron transport }\end{array}$ \\
\hline 11131 & CAPN11 & $\begin{array}{l}\text { Remodeling of cytoskeletal attachments to the plasma membrane during cell fusion and cell motility, pro- } \\
\text { teolytic modification of molecules in signal transduction pathways, degradation of enzymes controlling } \\
\text { progression through the cell cycle, regulation of gene expression, substrate degradation in some apoptotic } \\
\text { pathways, and an involvement in long-term potentiation }\end{array}$ \\
\hline 11142 & PKIG & $\begin{array}{l}\text { PKA inhibitors; protein kinase A has several functions in the cell, including regulation of glycogen, sugar, and } \\
\text { lipid metabolism }\end{array}$ \\
\hline 11252 & PACSIN2 & Involved in linking the actin cytoskeleton with vesicle formation by regulating tubulin polymerization \\
\hline 11332 & ACOT7 & $\begin{array}{l}\text { Catalyze the hydrolysis of acyl-CoAs to the free fatty acid and coenzyme A }(\mathrm{CoASH}) \text {, providing the potential } \\
\text { to regulate intracellular levels of acyl-CoAs, free fatty acids, and CoASH }\end{array}$ \\
\hline 23243 & ANKRD28 & Involved in the recognition of phosphoprotein substrates \\
\hline 23325 & KIAA1033 & Plays a key role in the fission of tubules that serve as transport intermediates during endosome sorting \\
\hline 23558 & WBP2 & Involved in mediating protein-protein interactions through the binding of polyproline ligands \\
\hline 26100 & WIPI2 & $\begin{array}{l}\text { Probable early component of the autophagy machinery being involved in formation of preautophagosomal } \\
\text { structures and their maturation into mature phagosomes }\end{array}$ \\
\hline 26505 & CNNM3 & Probable metal transporter \\
\hline 26520 & TIMM9 & Mediate the import and insertion of hydrophobic membrane proteins into the mitochondrial inner membrane \\
\hline 27075 & TSPAN13 & $\begin{array}{l}\text { Mediate signal transduction events that play a role in the regulation of cell development, activation, growth, } \\
\text { and motility }\end{array}$ \\
\hline 29105 & C16orf80 & $? ? ?$ \\
\hline 29927 & SEC61A1 & Plays a crucial role in the insertion of secretory and membrane polypeptides into the ER \\
\hline 50640 & PNPLA8 & Phospholipases which catalyze the cleavage of fatty acids from membrane phospholipids \\
\hline 51094 & ADIPOR1 & Regulates fatty acid catabolism and glucose levels \\
\hline 51491 & NOP16 & Involved in ribosome biogenesis \\
\hline 51504 & TRMT112 & Participates in both methylation of protein and tRNA species \\
\hline 51523 & CXXC5 & $\begin{array}{l}\text { Required for DNA damage-induced ATM phosphorylation, p53 activation, and cell cycle arrest. Involved in } \\
\text { myelopoiesis }\end{array}$ \\
\hline 51706 & CYB5R1 & $\begin{array}{l}\text { Involved in desaturation and elongation of fatty acids, cholesterol biosynthesis, drug metabolism, and, in } \\
\text { erythrocyte, methemoglobin reduction }\end{array}$ \\
\hline 54187 & NANS & Functions in the biosynthetic pathways of sialic acids \\
\hline 54606 & DDX56 & $\begin{array}{l}\text { Implicated in a number of cellular processes involving alteration of RNA secondary structure such as transla- } \\
\text { tion initiation, nuclear and mitochondrial splicing, and ribosome and spliceosome assembly. May play a role } \\
\text { in later stages of the processing of the preribosomal particles, leading to mature } 60 \text { S ribosomal subunits. Has } \\
\text { intrinsic ATPase activity }\end{array}$ \\
\hline 54941 & RNF125 & E3 ubiquitin-protein ligase that acts as a positive regulator of $\mathrm{T}$ cell activation \\
\hline 55062 & WIPI1 & May play a role in autophagy \\
\hline 55111 & PLEKHJ1 & ??? phospholipid binding \\
\hline 55238 & SLC38A7 & Mediates sodium-dependent transport of amino acids \\
\hline 55315 & SLC29A3 & Plays a role in cellular uptake of nucleosides, nucleobases, and their related analogs \\
\hline 55647 & RAB20 & Plays a role in the maturation and acidification of phagosomes that engulf pathogens \\
\hline 55700 & MAP7D1 & Mitotic spindle protein and member of the MAP7 (microtubule-associated protein 7) family of proteins \\
\hline 55743 & CHFR & $\begin{array}{l}\text { Functions in the antephase checkpoint by actively delaying passage into mitosis in response to microtubule } \\
\text { poisons }\end{array}$ \\
\hline 55898 & UNC45A & $\begin{array}{l}\text { Plays a role in cell proliferation and myoblast fusion, binds progesterone receptor and HSP90, and acts as a } \\
\text { regulator of the progesterone receptor chaperoning pathway }\end{array}$ \\
\hline 56005 & C19orf10 & $? ? ?$ \\
\hline 56267 & $C C B L 2$ & Encodes an aminotransferase that transaminates kynurenine to form kynurenic acid \\
\hline 56910 & STARD7 & $? ? ?$ \\
\hline 57409 & MIF4GD & Functions in replication-dependent translation of histone mRNAs \\
\hline 64754 & SMYD3 & Histone methyltransferase \\
\hline
\end{tabular}


Table 1 continued

\begin{tabular}{|c|c|c|}
\hline EntrezGeneID & Gene name & Functional description according to GeneCards \\
\hline 64787 & EPS8L2 & $\begin{array}{l}\text { Is thought to link growth factor stimulation to actin organization, generating functional redundancy in the } \\
\text { pathways that regulate actin cytoskeletal remodeling }\end{array}$ \\
\hline 64949 & MRPS26 & $\begin{array}{l}\text { Mammalian mitochondrial ribosomal proteins are encoded by nuclear genes and help in protein synthesis } \\
\text { within the mitochondrion }\end{array}$ \\
\hline 66036 & MTMR9 & Thought to have a role in the control of cell proliferation \\
\hline 79056 & PRRG4 & ??? Calcium ion binding \\
\hline 80227 & PAAF1 & Involved in regulation of association of proteasome components \\
\hline 80775 & TMEM177 & $? ? ?$ \\
\hline 89870 & TRIM15 & $? ? ?$ \\
\hline 91663 & MYADM & $\begin{array}{l}\text { ??? Regulates the connection between the plasma membrane and the cortical cytoskeleton and so can control } \\
\text { the endothelial inflammatory response }\end{array}$ \\
\hline 114971 & PTPMT1 & $\begin{array}{l}\text { Is an essential intermediate in the biosynthetic pathway of cardiolipin, a mitochondrial-specific phospholipid } \\
\text { regulating the membrane integrity and activities of the organelle }\end{array}$ \\
\hline 124583 & CANT1 & Functions as a calcium-dependent nucleotidase \\
\hline 127687 & C1orf122 & $? ? ?$ \\
\hline 135932 & TMEM139 & $? ? ?$ \\
\hline 140465 & MYL6B & Regulatory light chain of myosin \\
\hline 140606 & SELM & May function as a thiol-disulfide-oxidoreductase that participates in disulfide bond formation \\
\hline 147007 & TMEM199 & $? ? ?$ \\
\hline 151613 & TTC14 & ??? RNA binding \\
\hline 155066 & ATP6V0E2 & $\begin{array}{l}\text { Play an important role in processes such as receptor-mediated endocytosis, protein degradation, and coupled } \\
\text { transport }\end{array}$ \\
\hline 196383 & RILPL2 & $\begin{array}{l}\text { Involved in cell shape and neuronal morphogenesis, positively regulating the establishment and maintenance } \\
\text { of dendritic spines }\end{array}$ \\
\hline 252839 & TMEM9 & May be involved in intracellular transport \\
\hline 253461 & ZBTB38 & May be involved in the differentiation and/or survival of late postmitotic neurons \\
\hline 375757 & C9orf119 & Required for double-strand break repair via homologous recombination \\
\hline 389203 & C4orf52 & $? ? ?$ \\
\hline 100128750 & LOC100128750 & $? ? ?$ \\
\hline 100505687 & LOC100505687 & $? ? ?$ \\
\hline
\end{tabular}

The description of the functionality of the gene has been taken from GeneCards; genes involved in mitochondrial processes according to the MITOP2 database are in italic

the variability in metabolite level in these same donors, a Pearson-based correlation analysis between the top $1 \%$ variable genes and all metabolites was performed (cutoff $R_{2}>0.7$ ). Out of the 99 most variable genes, 91 could be linked to the variation in metabolites on an individual level, meaning that these 91 genes can at least partially explain the interindividual variation observed in metabolites. In particular, hydroxy-APAP, methoxy-APAP, and the tentatively identified metabolite $\mathrm{C}_{8} \mathrm{H}_{13} \mathrm{O}_{5} \mathrm{~N}$-APAP-glucuronide showed strong correlations with genes on an individual level ( $n=36,36$, and 51 correlating genes, respectively). Interestingly, $\mathrm{C}_{8} \mathrm{H}_{13} \mathrm{O}_{5} \mathrm{~N}$-APAP-glucuronide has previously been reported by Jetten et al. (2012) as a novel APAP metabolite, which could be detected in the in vivo human situation after low-dose APAP exposure. This metabolite could thus be confirmed in the current study in an in vitro human situation consisting of primary human hepatocytes.
Furthermore, a mass tentatively assigned to 3,3'-biacetaminophen (not detected previously) has also been found. 3,3'-biacetaminophen has been suggested to result from NAPQI reacting with APAP and is considered a reactive oxygen species (ROS) product (Chen et al. 2008a).

\section{Discussion}

The aim of this study is to evaluate the interindividual differences in gene expression changes and APAP metabolite formation in primary human hepatocytes of several donors $(n=5)$ exposed to a non-toxic to toxic APAP dose range.

Interindividual variation in gene expression is a very common phenomenon; therefore, we have focused on the gene expression changes that are most different between individuals in response to APAP exposure. To do so, we 


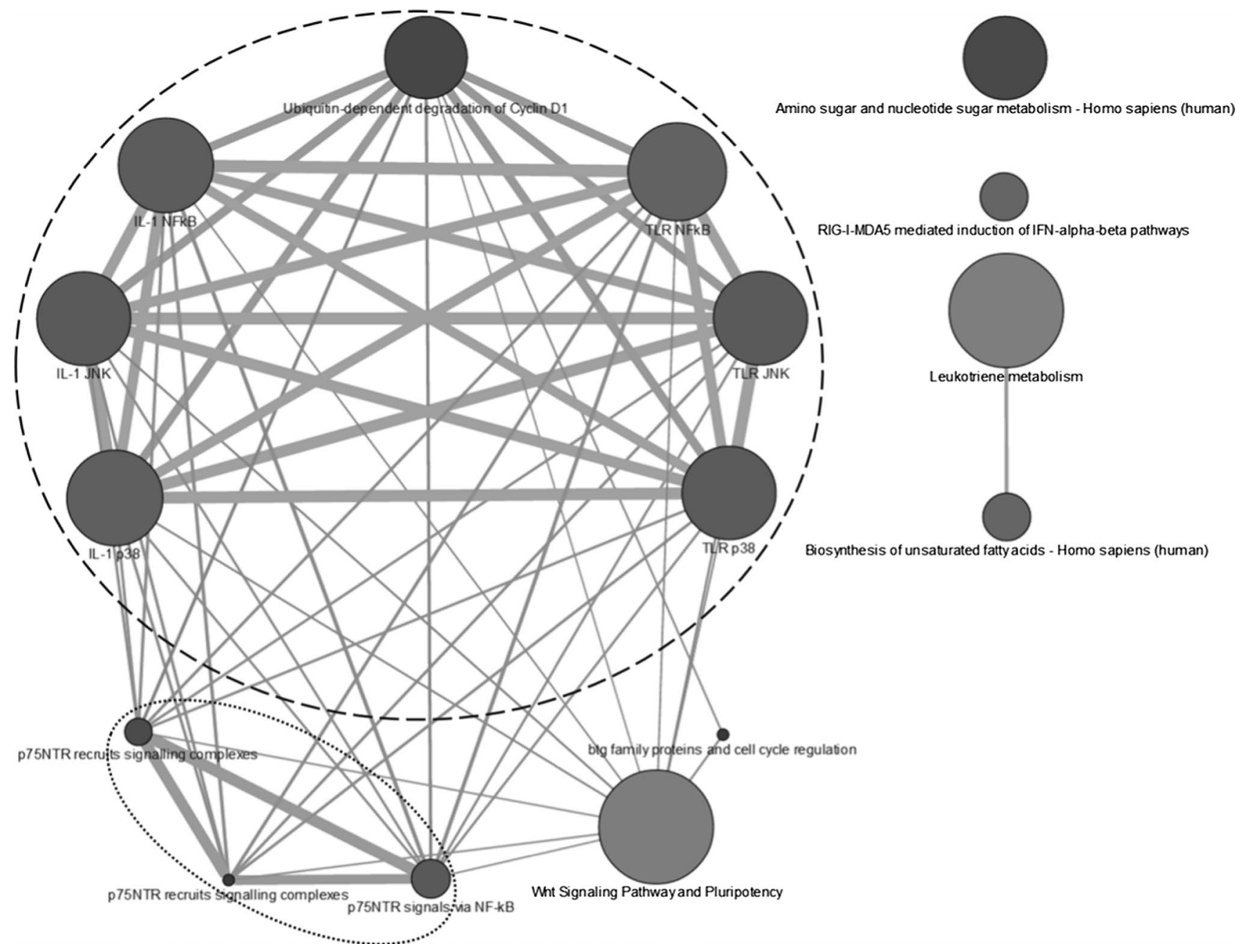

Fig. 1 Network of the top $1 \%$ most variable genes between donors after APAP exposure. The network was created based on a gene set overrepresentation analysis (ConsensusPathDB). Each node represents a biological pathway, the size of the node represents the amount of genes included in the pathway (bigger diameter = larger \# genes), the color of the node represents its significance (darker gray = lower $p$ value), and the thickness of the edge represents the amount of overlap between the connected nodes (thicker line $=$ higher \# overlapping genes)
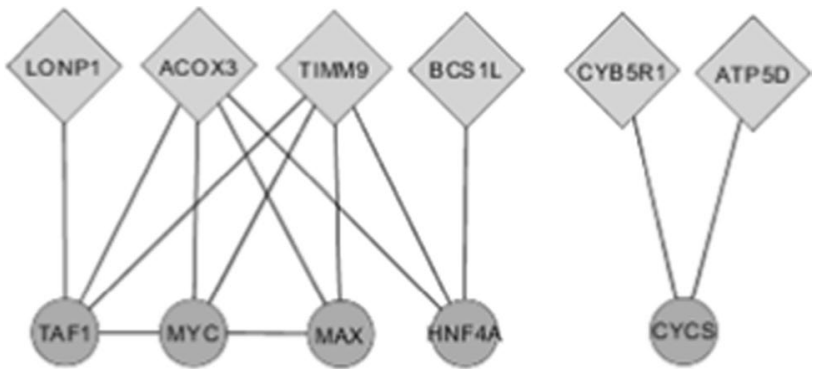

Fig. 2 Network of highly variable mitochondrial-related genes. Nearest neighbor analysis was performed on all mitochondrial-related genes from the top $1 \%$ highly variable gene list. Only the nearest neighbors shared by more than one of the variable genes were taken into account. Square nodes represent input genes, and round nodes represent the shared nearest neighbors have created a short list consisting of the top $1 \%$ most different genes based on correlation analysis $(n=99$, see Table 1). Expression levels of many genes/metabolites including, but not limited to cytochrome P450 enzymes, glucuronosyltransferases, sulfotransferases, and glutathione S-transferases have been shown to influence the biotransformation processes of APAP (Zhao and Pickering 2011). However, studies in general link baseline expression levels of these genes to APAP metabolism parameters, while in the current study we focus on response parameters after APAP exposure in order to explain interindividual variability.

To define the biological functionality of the genes with the highest variability between individuals (top $1 \%$ list), 
Fig. 3 Schematic visualization of APAP metabolic pathway. The log-transformed metabolite levels for each donor on at each dose corrected for $0 \mathrm{mM}$ are visualized. Gray boxes not measured/detected. Increase in a metabolite is pictured from green (no increase, equals a numerical value of 0 on a $\log$ scale) to yellow, orange, and red (high increase, maximum value $=5$ on a $\log$ scale). Figure adapted from Jetten et al. (2012) (color figure online)

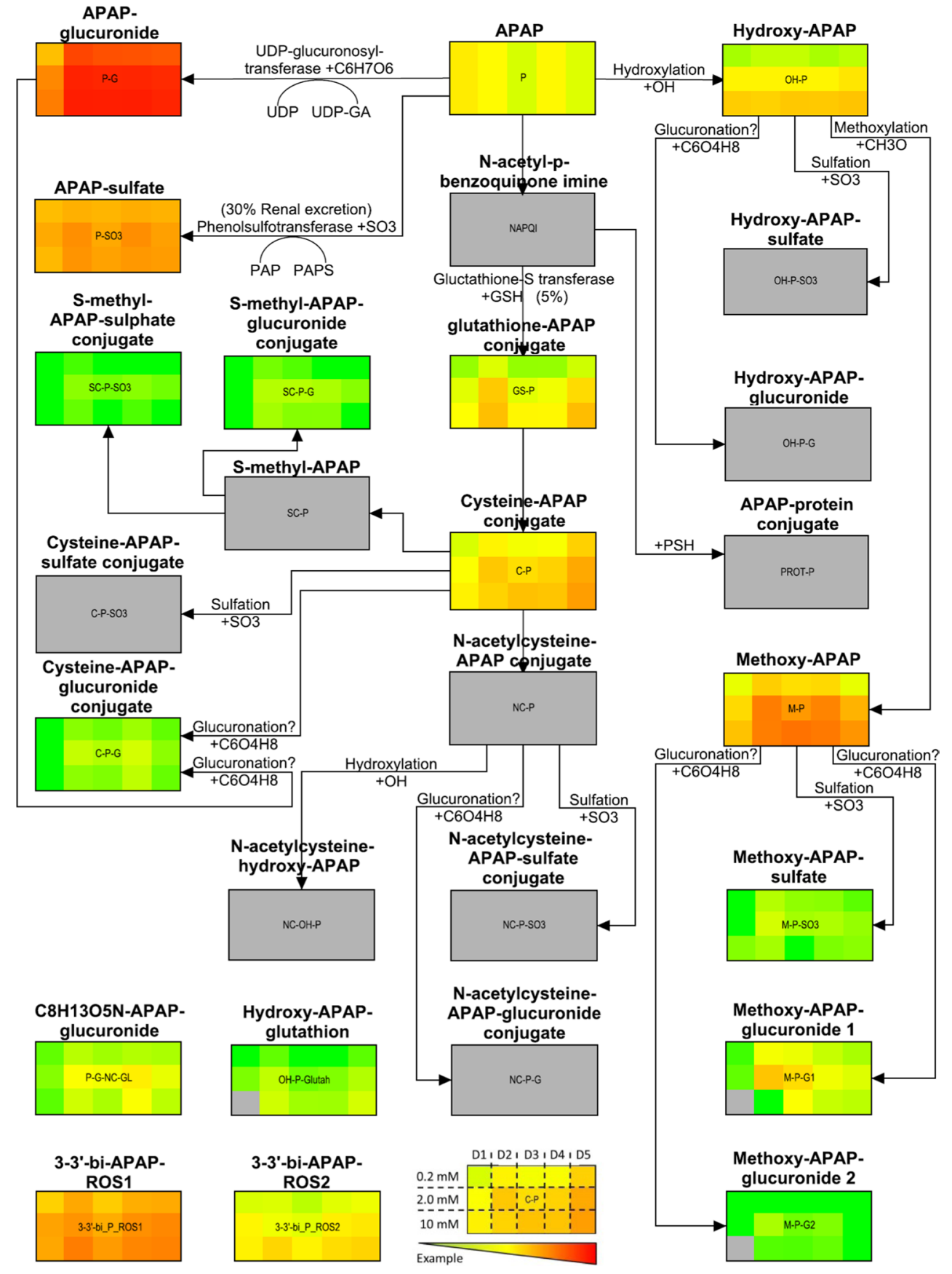

a network of pathways found by gene set overrepresentation analysis on this list was created (Fig. 1). This network shows a large cluster with TLRs, JNK, NF-кB, IL-1, p38, and cyclin D1-related pathways (encircled with striped line). TLR, JNK, and NF- $\mathrm{B}$ pathways are all key regulators in the production of cytokines associated with inflammatory responses and the early stages of the development of hepatocarcinogenesis (Maeda 2010). Furthermore, all of the above-mentioned pathways have been associated with the process of liver regeneration (Iimuro and Fujimoto 2010). Hepatocytes rarely undergo proliferation in the liver under normal circumstances. However, proliferation can be triggered in response to loss of liver mass for instance induced by liver resection but also due to toxininduced hepatocyte trauma like is the case with APAP. In both in vitro and in vivo studies, APAP has been shown to induce a persistent activation of JNK adding to hepatocellular necrosis (Gunawan et al. 2006). Cross talk between JNK and NF- $\mathrm{B}$ has been proposed as a mechanism through which JNK mediates cell death. Matsumura et al. (2003) showed that inhibition of NF- $\kappa$ B in the liver by APAP leads to amplification of JNK and as such shifts the 
balance from cell survival toward cell death. IL-1 stimulates both the JNK and NF- $\mathrm{KB}$ pathways, which increases cell signaling and interferes with the cell cycle (Matsuzawa et al. 2005; Sanz-Garcia et al. 2013). TLR plays a role in the expression of cytokines and hepatomitogens; in response to TRL activation, p38 is triggered, leading to cytokine- and stress-induced apoptosis (Matsuzawa et al. 2005; Sanz-Garcia et al. 2013). Finally, it is indicated that that NF- $\mathrm{BB}$ activation operates on cell growth through cyclin D1 expression (Guttridge et al. 1999; Maeda 2010). In acetaminophen-induced liver injury, sustained JNK activation, through NF- $\mathrm{KB}$, is essential in inducing apoptosis (Hanawa et al. 2008). Furthermore, it is suggested that NAPQI-induced damage on its own is not enough to cause hepatocyte death after APAP dosing and that activation of signaling pathways involving JNK is necessary to lead to cell death (Hanawa et al. 2008; Kaplowitz 2005). The actual downstream targets of JNK that are involved in APAP-induced liver injury are still largely unknown; however, a role for mitochondrial proteins has recently been suggested (Zhou et al. 2008).

Interestingly, $10 \%$ of the genes from the top $1 \%$ highly variable gene list consist of mitochondrial-related genes (see Table 1; genes in Italic). The majority of these genes are involved in metabolism-related processes [CCBL2 (Yu et al. 2006), PTPMT1 (Shen et al. 2011), ACOX3 (Cui et al. 2010), ACOT7 (Fujita et al. 2011), and CYB5R1 (Chae et al. 2013)], while others are part of the respiratory chain complexes in the mitochondria [ATP5D (Sotgia et al. 2012) and BCS1L (Kotarsky et al. 2012)] or are involved in more structurally-related processes like protein synthesis [MRPS26 (Sotgia et al. 2012)] and mitochondrial matrix or membrane maintenance [LONP1 (Tian et al. 2011) and TIMM9 (Sotgia et al. 2012), respectively]. All but one (MRPS26) of the above-mentioned genes has been related to the toxic/necrotic effects of APAP in a study comparing the toxicity response to APAP in rats/mice with the response to APAP's far less toxic stereo-isomer N-acetylm-aminophenol [AMAP; (Beyer et al. 2007)]. In addition, ATP5D, MRPS26, LONP1, ACOT7, and TIMM9 were also shown to be regulated in HepG2 cells exposed to a toxic (10 mM) APAP dose for 12-72 h (unpublished results). Drug-induced liver injury has often been linked to regulation of the mitochondrial stress responses, which include the formation of ROS products, alterations in lipid metabolism, electron transport, cofactor metabolism, and the activation of pathways important in determining cell survival or death (Beyer et al. 2007; Han et al. 2013).

The nearest neighbor analyses on all mitochondrialrelated genes from the top $1 \%$ highly variable gene list show that mainly transcription factors seem to be the binding element in the response of the mitochondrial-related genes (Fig. 2). The involvement of transcription factors has been suggested in the drug-induced stress response of mitochondria in hepatocytes (Han et al. 2013). This indicates that interindividual variation exists in the response of mitochondrial-related genes, possibly explaining part of the differences between humans in mitochondrial-related APAP-induced toxicity responses and consequential liver damage levels.

Furthermore, in the gene set overrepresentation network, another smaller network around p75NTR is present (Fig. 1, encircled with dotted line). P75 NTR is a cell membrane receptor protein that has been associated with tumor and metastasis suppression (Khwaja et al. 2004), similar to the cluster described above. Non-steroidal anti-inflammatory drugs (NSAIDs) are used to reduce inflammation and also act as analgesics by the inhibition of cyclooxygenase-2 (COX-2). However, high concentrations of some NSAIDs are able to reduce proliferation and induce apoptosis in cancer cells. Several molecular mechanisms have been proposed as possible mediators in the anticancerous effects of NSAIDs, including p75NTR. Although APAP is not considered to be a real NSAID, due to its limited antiinflammatory effects, APAP does affect similar pathways and works as an analgesic through COX-2 inhibition which might explain why similar effects have been suggested for APAP (Bonnefont et al. 2007).

Two other components in the gene set overrepresentation network that are connected to the components described above are the 'Wnt-signaling pathway and pluripotency' and 'BTG family proteins and cell cycle regulation' (see Fig. 1). Both pathways can be related to APAPinduced toxicity-related effects. The stimulation of the Wnt pathway has been suggested to be beneficial after APAPinduced liver failure by stimulating liver regeneration (Apte et al. 2009). This corresponds with the previously mentioned regulation of liver regeneration by the genes involved in the cluster around TLRs, JNK, NF-кB, IL-1, p38, and cyclin D1-related pathways. The BGT gene family has been associated with APAP hepatotoxicity before (Beyer et al. 2007) and has a function in DNA-strand break repair (Choi et al. 2012) and in the regulation of reactive oxygen species generation in the mitochondria (Lim et al. 2012). Both APAP and NAPQI are known to covalently bind to DNA and cause DNA damage (Dybing et al. 1984; Rannug et al. 1995), which possibly explains why this pathway is triggered by APAP exposure. As already described above, mitochondrial stress is an intricate part of the cellular response to APAP-induced oxidative stress (Hanawa et al. 2008), which might also explain the response of the BTG-related pathway. These findings agree with the previously mentioned interindividual variation in mitochondrial genes.

In addition, several other pathways not connected to the main cluster of pathways are included in the network of the 
gene set overrepresentation analysis. These are 'leukotriene metabolism' together with 'biosynthesis of unsaturated fatty acids,' 'amino sugar and nucleotide sugar metabolism' and 'RIG-I-MDA5-mediated induction of IFN-alpha-beta.' The first set of pathways (leukotrienes and fatty acids) probably represents the variation in the normal, therapeutic mechanism of APAP. In humans, unsaturated fatty acids are bioactivated through enzymatic oxygenation to, among others, prostaglandins and leukotrienes which contribute to fever, pain, inflammation, and cancer development (Ricciotti and FitzGerald 2011). APAP interferes with these processes as such inhibiting symptoms. Concerning the sugar metabolism pathway, carbohydrate homeostasis is essential for normal liver function. It is well known that during APAP-induced liver failure, these processes are severely affected (Record et al. 1975). Finally, RIG-I-MDA5-mediated induction of IFN-alpha-beta is a process that has been linked to several liver pathologies like hepatitis $\mathrm{A} / \mathrm{B} / \mathrm{C}$ and hepatic steatosis (Kawai et al. 2005; Toyoda et al. 2013; Wei et al. 2010). Although no apparent link with APAP is available in the literature, it seems that this process is somehow linked to an APAP-induced stress response.

To determine how the variation in the above-mentioned genes can explain the interindividual variation in metabolism levels, a correlation analysis was performed between the top $1 \%$ most variable genes and all metabolites. Glucuronidation and sulfation are the two major processes in APAP metabolism, resulting in APAP-glucuronide and APAP-sulfate, respectively, which are non-toxic APAP metabolites (see Fig. 3; Chen et al. 2008a). In addition, another less abundant route of APAP metabolism utilizes hydroxylation/methoxylation of APAP. Hydroxy-APAP and methoxy-APAP are oxidative metabolites formed during this route of APAP metabolism, and both these metabolites have been associated with the hepatotoxic effects of APAP (Chen et al. 2008a; Dahlin et al. 1984b; Wilson et al. 1982). The variation in both these metabolites could be explained by a large proportion of the genes from the top $1 \%$ most variable genes ( $n=36$ for both metabolites). It thus seems that the largest interindividual variation in gene expression responses after APAP exposure can be linked to the formation of toxic APAP metabolite formation. Interestingly, hydroxy/methoxy-derived metabolites could also be detected in human in vivo low-dose APAP exposure (Jetten et al. 2012). The fact that these metabolites and their derivatives are detectable both in vivo and in vitro, even at APAP doses within the therapeutic range and that their variation in expression levels between individuals can be linked to the variation in gene expression of genes related to toxicity-related effects of APAP exposure, indicates a role as potential key elements in the molecular mechanism behind APAP toxicity. Additionally, another metabolite, $\mathrm{C}_{8} \mathrm{H}_{13} \mathrm{O}_{5} \mathrm{~N}$-APAP-glucuronide, also shows correlation with a large set of the top $1 \%$ variable genes $(n=51)$. This metabolite was described as new in the low-dose in vivo APAP exposure study of Jetten et al. (2012). Since this metabolite is relatively unknown, further studies on its exact route of metabolism and toxic potency could lead to further insight into the molecular mechanism behind APAP toxicity for the same reasons as explained for hydroxyl/ methoxy-APAP above.

In summary, the biological processes in which the genes with the highest variability in expression between individuals after APAP exposure are involved can be linked to APAP-toxicity-related processes like liver regeneration, inflammatory responses, and mitochondrial stress responses. Also, processes related to hepatocarcinogenesis, cell cycle, and drug efficacy show large interindividual variation after APAP exposure.

In addition, most of the genes with high variability between individuals after APAP exposure can be linked to variability in expression levels of metabolites (hydroxyl/ methoxy-APAP and C8H13O5N-APAP-glucuronide). Possibly, these findings could help explain the differences seen in susceptibility to APAP toxicity in the in vivo situation. Furthermore, they might give an indication for where the factors causing variability in susceptibility toward APAPinduced liver failure could be found.

Acknowledgments This work was supported by the Dutch Ministry of Public Health, Welfare and Sports (VWS) as a part of the Assuring Safety without Animal Testing (ASAT) initiative.

Conflict of interest The authors declare that they have no conflict of interest.

Open Access This article is distributed under the terms of the Creative Commons Attribution 4.0 International License (http://creativecommons.org/licenses/by/4.0/), which permits unrestricted use, distribution, and reproduction in any medium, provided you give appropriate credit to the original author(s) and the source, provide a link to the Creative Commons license, and indicate if changes were made.

\section{References}

Apte U, Singh S, Zeng G et al (2009) Beta-catenin activation promotes liver regeneration after acetaminophen-induced injury. Am J Pathol 175(3):1056-1065. doi:10.2353/ajpath.2009.080976

Bannwarth B, Pehourcq F, Lagrange F et al (2001) Single and multiple dose pharmacokinetics of acetaminophen (paracetamol) in polymedicated very old patients with rheumatic pain. J Rheumatol 28(1):182-184

Berg EL (2014) Systems biology in drug discovery and development. Drug Discov Today 19(2):113-125. doi:10.1016/j. drudis.2013.10.003

Beyer RP, Fry RC, Lasarev MR et al (2007) Multicenter study of acetaminophen hepatotoxicity reveals the importance of biological endpoints in genomic analyses. Toxicol Sci 99(1):326-337. doi:10.1093/toxsci/kfm150 
Bonnefont J, Daulhac L, Etienne M et al (2007) Acetaminophen recruits spinal p42/p44 MAPKs and GH/IGF-1 receptors to produce analgesia via the serotonergic system. Mol Pharmacol 71(2):407-415. doi:10.1124/mol.106.025775

Borin MT, Ayres JW (1989) Single dose bioavailability of acetaminophen following oral administration. Int J Pharm 54(3):199-209

Chae YC, Angelin A, Lisanti S et al (2013) Landscape of the mitochondrial Hsp90 metabolome in tumours. Nat Commun 4:2139. doi: $10.1038 /$ ncomms 3139

Chen C, Krausz KW, Idle JR, Gonzalez FJ (2008a) Identification of novel toxicity-associated metabolites by metabolomics and mass isotopomer analysis of acetaminophen metabolism in wildtype and Cyp2e1-null mice. J Biol Chem 283(8):4543-4559. doi:10.1074/jbc.M706299200

Chen C, Krausz KW, Idle JR, Gonzalez FJ (2008b) Identification of novel toxicity-associated metabolites by metabolomics and mass isotopomer analysis of acetaminophen metabolism in wildtype and Cyp2e1-null mice. J Biol Chem 283(8):4543-4559. doi:10.1074/jbc.M706299200

Choi KS, Kim JY, Lim SK et al (2012) TIS21(/BTG2/PC3) accelerates the repair of DNA double strand breaks by enhancing Mre11 methylation and blocking damage signal transfer to the Chk 2(T68)-p53(S20) pathway. DNA Repair (Amst) 11(12):965-975. doi:10.1016/j.dnarep.2012.09.009

Court MH, Duan SX, von Moltke LL et al (2001) Interindividual variability in acetaminophen glucuronidation by human liver microsomes: identification of relevant acetaminophen UDP-glucuronosyltransferase isoforms. J Pharmacol Exp Ther 299(3):998-1006

Critchley JAJH, Critchley LAH, Anderson PJ, Tomlinson B (2005) Differences in the single-oral-dose pharmacokinetics and urinary excretion of paracetamol and its conjugates between Hong Kong Chinese and Caucasian subjects. J Clin Pharm Ther 30(2):179184. doi:10.1111/j.1365-2710.2004.00626.x

Cui Z, Hou J, Chen X et al (2010) The profile of mitochondrial proteins and their phosphorylation signaling network in INS-1 beta cells. J Proteome Res 9(6):2898-2908. doi:10.1021/pr100139z

Dahlin DC, Miwa GT, Lu AY, Nelson SD (1984a) $N$-acetyl-p-benzoquinone imine: a cytochrome P-450-mediated oxidation product of acetaminophen. Proc Natl Acad Sci USA 81(5):1327-1331

Dahlin DC, Miwa GT, Lu AY, Nelson SD (1984b) N-acetyl-p-benzoquinone imine: a cytochrome P-450-mediated oxidation product of acetaminophen. Proc Natl Acad Sci USA 81(5):1327-1331

Dai M, Wang P, Boyd AD et al (2005) Evolving gene/transcript definitions significantly alter the interpretation of GeneChip data. Nucleic Acids Res 33(20):e175. doi:10.1093/nar/gni179

Daykin CA, Foxall PJ, Connor SC, Lindon JC, Nicholson JK (2002) The comparison of plasma deproteinization methods for the detection of low-molecular-weight metabolites by (1) H nuclear magnetic resonance spectroscopy. Anal Biochem 304(2):220 230. doi:10.1006/abio.2002.5637

Douglas DR, Sholar JB, Smilkstein MJ (1996) A pharmacokinetic comparison of acetaminophen products (tylenol extended relief vs regular tylenol). Acad Emerg Med 3(8):740-744

Dybing E, Holme JA, Gordon WP, Soderlund EJ, Dahlin DC, Nelson SD (1984) Genotoxicity studies with paracetamol. Mutat Res 138(1):21-32

Eijssen LM, Jaillard M, Adriaens ME et al (2013) User-friendly solutions for microarray quality control and pre-processing on ArrayAnalysis.org. Nucleic Acids Res 41(Web Server issue):W71W76. doi:10.1093/nar/gkt293

Elstner M, Andreoli C, Klopstock T, Meitinger T, Prokisch H (2009) The mitochondrial proteome database: MitoP2. Methods Enzymol 457:3-20. doi:10.1016/s0076-6879(09)05001-0

Fisher MB, Vandenbranden M, Findlay K et al (2000) Tissue distribution and interindividual variation in human UDP-glucuronosyltransferase activity: relationship between UGT1A1 promoter genotype and variability in a liver bank. Pharmacogenetics 10(8):727-739

Fujita M, Momose A, Ohtomo T et al (2011) Upregulation of fatty acyl-CoA thioesterases in the heart and skeletal muscle of rats fed a high-fat diet. Biol Pharm Bull 34(1):87-91

Gao J, Ade AS, Tarcea VG et al (2009) Integrating and annotating the interactome using the MiMI plugin for cytoscape. Bioinformatics 25(1):137-138. doi:10.1093/bioinformatics/btn501

Greek R, Menache A (2013) Systematic reviews of animal models: methodology versus epistemology. Int J Med Sci 10(3):206-221. doi:10.7150/ijms.5529

Gunawan BK, Liu ZX, Han D, Hanawa N, Gaarde WA, Kaplowitz $\mathrm{N}$ (2006) c-Jun N-terminal kinase plays a major role in murine acetaminophen hepatotoxicity. Gastroenterology 131(1):165178. doi:10.1053/j.gastro.2006.03.045

Guttridge DC, Albanese C, Reuther JY, Pestell RG, Baldwin AS Jr (1999) NF-kappaB controls cell growth and differentiation through transcriptional regulation of cyclin D1. Mol Cell Biol 19(8):5785-5799

Han D, Dara L, Win S et al (2013) Regulation of drug-induced liver injury by signal transduction pathways: critical role of mitochondria. Trends Pharmacol Sci 34(4):243-253. doi:10.1016/j. tips.2013.01.009

Hanawa N, Shinohara M, Saberi B, Gaarde WA, Han D, Kaplowitz N (2008) Role of JNK translocation to mitochondria leading to inhibition of mitochondria bioenergetics in acetaminopheninduced liver injury. J Biol Chem 283(20):13565-13577. doi:10.1074/jbc.M708916200

Hinson JA, Roberts DW, James LP (2010) Mechanisms of acetaminophen-induced liver necrosis. Handb Exp Pharmacol 196:369405. doi:10.1007/978-3-642-00663-0_12

Iimuro Y, Fujimoto J (2010) TLRs, NF-kB, JNK, and liver regeneration. Gastroenterol Res Pract. doi:10.1155/2010/598109

Invitrogen ${ }^{\mathrm{TM}}$ hepatic biology products and services (2009) No standard like a gold standard, pp 11-15. http://tools.lifetechnologies. com/content/sfs/brochures/ADMEToxGoldStandard.pdf

Jaeschke H, McGill MR, Ramachandran A (2012) Oxidant stress, mitochondria, and cell death mechanisms in drug-induced liver injury: lessons learned from acetaminophen hepatotoxicity. Drug Metab Rev 44(1):88-106

Jetten MJ, Gaj S, Ruiz-Aracama A et al (2012) Omics analysis of low dose acetaminophen intake demonstrates novel response pathways in humans. Toxicol Appl Pharmacol 259(3):320-328. doi:10.1016/j.taap.2012.01.009

Kamali F (1993) The effect of probenecid on paracetamol metabolism and pharmacokinetics. Eur J Clin Pharmacol 45(6):551-553. doi:10.1007/bf00315313

Kamburov A, Stelzl U, Lehrach H, Herwig R (2013) The ConsensusPathDB interaction database: 2013 update. Nucleic Acids Res 41(Database issue):D793-D800. doi:10.1093/nar/gks1055

Kaplowitz N (2005) Idiosyncratic drug hepatotoxicity. Nat Rev Drug Discov 4(6):489-499. doi:10.1038/nrd1750

Kawai T, Takahashi K, Sato S et al (2005) IPS-1, an adaptor triggering RIG-I- and Mda5-mediated type I interferon induction. Nat Immunol 6(10):981-988. doi:10.1038/ni1243

Khwaja F, Allen J, Lynch J, Andrews P, Djakiew D (2004) Ibuprofen inhibits survival of bladder cancer cells by induced expression of the p75NTR tumor suppressor protein. Cancer Res 64(17):62076213. doi:10.1158/0008-5472.CAN-03-3814

Kienhuis AS, van de Poll MC, Wortelboer H et al (2009) Parallelogram approach using rat-human in vitro and rat in vivo toxicogenomics predicts acetaminophen-induced hepatotoxicity in humans. Toxicol Sci 107(2):544-552. doi:10.1093/toxsci/ kfn237

Kotarsky H, Keller M, Davoudi M et al (2012) Metabolite profiles reveal energy failure and impaired beta-oxidation in liver of mice 
with complex III deficiency due to a BCS1L mutation. PLoS ONE 7(7):e41156. doi:10.1371/journal.pone.0041156

Lee WM (2007) Acetaminophen toxicity: changing perceptions on a social/medical issue. Hepatology 46(4):966-970. doi:10.1002/ hep. 21926

Lim SK, Choi YW, Lim IK, Park TJ (2012) BTG2 suppresses cancer cell migration through inhibition of Src-FAK signaling by downregulation of reactive oxygen species generation in mitochondria. Clin Exp Metastasis 29(8):901-913. doi:10.1007/ s10585-012-9479-z

Lommen A (2009) MetAlign: interface-driven, versatile metabolomics tool for hyphenated full-scan mass spectrometry data preprocessing. Anal Chem 81(8):3079-3086. doi:10.1021/ ac900036d

Lommen A, Gerssen A, Oosterink JE et al (2011) Ultra-fast searching assists in evaluating sub-ppm mass accuracy enhancement in U-HPLC/Orbitrap MS data. Metabolomics 7(1):15-24. doi:10.1007/s11306-010-0230-y

Maeda S (2010) NF-kappaB, JNK, and TLR signaling pathways in hepatocarcinogenesis. Gastroenterol Res Pract 2010:367694. doi: $10.1155 / 2010 / 367694$

Matsumura T, Degawa T, Takii T et al (2003) TRAF6-NF-kappaB pathway is essential for interleukin-1-induced TLR2 expression and its functional response to TLR2 ligand in murine hepatocytes. Immunology 109(1):127-136

Matsuzawa A, Saegusa K, Noguchi T et al (2005) ROS-dependent activation of the TRAF6-ASK1-p38 pathway is selectively required for TLR4-mediated innate immunity. Nat Immunol 6(6):587-592. doi:10.1038/ni1200

Polasek TM, Elliot DJ, Miners JO (2006) Measurement of human cytochrome P4501A2 (CYP1A2) activity in vitro. Curr Protoc Toxicol 4.19.1-4.19.11. doi:10.1002/0471140856.tx0419s27

Portolés A, Puerro M, Terleira A et al (2003) A new high-absorptionrate Paracetamol 500-mg formulation: a comparative bioavailability study in healthy volunteers. Curr Ther Res 64(7):401-411

Rannug U, Holme JA, Hongslo JK, Sram R (1995) International commission for protection against environmental mutagens and carcinogens. An evaluation of the genetic toxicity of paracetamol. Mutat Res 327(1-2):179-200

R-Core-Team (2013) R: a language and environment for statistical computing. $\mathrm{R}$ foundation for statistical computing. http:// www.R-project.org/

Record CO, Chase RA, Alberti KG, Williams R (1975) Disturbances in glucose metabolism in patients with liver damage due to paracetamol overdose. Clin Sci Mol Med 49(5):473-479

Ricciotti E, FitzGerald GA (2011) Prostaglandins and inflammation. Arterioscler Thromb Vasc Biol 31(5):986-1000. doi:10.1161/ ATVBAHA.110.207449

Ruiz-Aracama A, Peijnenburg A, Kleinjans J et al (2011) An untargeted multi-technique metabolomics approach to studying intracellular metabolites of $\mathrm{HepG} 2$ cells exposed to 2,3,7,8-tetrachlorodibenzo-p-dioxin. BMC Genomics 12:251. doi:10.1186/1471-2164-12-251

Rygnestad T, Zahlsen K, Samdal FA (2000) Absorption of effervescent paracetamol tablets relative to ordinary paracetamol tablets in healthy volunteers. Eur J Clin Pharmacol 56(2):141-143. doi:10.1007/s002280050732

Sabate M, Ibanez L, Perez E et al (2011) Paracetamol in therapeutic dosages and acute liver injury: causality assessment in a prospective case series. BMC Gastroenterol 11:80. doi:10.1186/1471-230x-11-80
Sanz-Garcia C, Ferrer-Mayorga G, Gonzalez-Rodriguez A et al (2013) Sterile inflammation in acetaminophen-induced liver injury is mediated by Cot/tpl2. J Biol Chem 288(21):1534215351. doi:10.1074/jbc.M112.439547

Shen J, Liu X, Yu WM et al (2011) A critical role of mitochondrial phosphatase Ptpmt1 in embryogenesis reveals a mitochondrial metabolic stress-induced differentiation checkpoint in embryonic stem cells. Mol Cell Biol 31(24):4902-4916. doi:10.1128/ MCB.05629-11

Sotgia F, Whitaker-Menezes D, Martinez-Outschoorn UE et al (2012) Mitochondria "fuel" breast cancer metabolism: fifteen markers of mitochondrial biogenesis label epithelial cancer cells, but are excluded from adjacent stromal cells. Cell Cycle 11(23):4390 4401. doi:10.4161/cc.22777

Tan C, Graudins A (2006) Comparative pharmacokinetics of Panadol Extend and immediate-release paracetamol in a simulated overdose model. Emerg Med Australas 18(4):398-403. doi:10.1111/j.1742-6723.2006.00873.x

Tian Q, Li T, Hou W, Zheng J, Schrum LW, Bonkovsky HL (2011) Lon peptidase 1 (LONP1)-dependent breakdown of mitochondrial 5 -aminolevulinic acid synthase protein by heme in human liver cells. J Biol Chem 286(30):26424-26430. doi:10.1074/jbc. M110.215772

Toyoda H, Kumada T, Kiriyama S et al (2013) Association between hepatic steatosis and hepatic expression of genes involved in innate immunity in patients with chronic hepatitis C. Cytokine 63(2):145-150. doi:10.1016/j.cyto.2013.04.012

Turkoski BB (2010) Acetaminophen: old friend-new rules. Orthop Nurs 29(1):41-43. doi:10.1097/NOR.0b013e3181c8cd75 (quiz 44-5)

van Iersel MP, Kelder T, Pico AR et al (2008) Presenting and exploring biological pathways with pathvisio. BMC Bioinform 9:399. doi:10.1186/1471-2105-9-399

Wei C, Ni C, Song T et al (2010) The hepatitis B virus X protein disrupts innate immunity by downregulating mitochondrial antiviral signaling protein. J Immunol 185(2):1158-1168. doi:10.4049/ jimmunol.0903874

Wilson JM, Slattery JT, Forte AJ, Nelson SD (1982) Analysis of acetaminophen metabolites in urine by high-performance liquid chromatography with UV and amperometric detection. J Chromatogr 227(2):453-462

Yasar U, Greenblatt DJ, Guillemette C, Court MH (2013) Evidence for regulation of UDP-glucuronosyltransferase (UGT) 1A1 protein expression and activity via DNA methylation in healthy human livers. J Pharm Pharmacol 65(6):874-883. doi:10.1111/ jphp. 12053

Yin OQP, Tomlinson B, Chow AHL, Chow MSS (2001) Pharmacokinetics of acetaminophen in Hong Kong Chinese subjects. Int $\mathbf{J}$ Pharm 222(2):305-308

Yu P, Li Z, Zhang L, Tagle DA, Cai T (2006) Characterization of kynurenine aminotransferase III, a novel member of a phylogenetically conserved KAT family. Gene 365:111-118. doi:10.1016/j.gene.2005.09.034

Zhao L, Pickering G (2011) Paracetamol metabolism and related genetic differences. Drug Metab Rev 43(1):41-52. doi:10.3109 /03602532.2010.527984

Zhou Q, Lam PY, Han D, Cadenas E (2008) c-Jun N-terminal kinase regulates mitochondrial bioenergetics by modulating pyruvate dehydrogenase activity in primary cortical neurons. J Neurochem 104(2):325-335. doi:10.1111/j.1471-4159.2007.04957.x 